\title{
TEMPERATURAS EXTREMAS E AS MUDANÇAS CLIMÁTICAS EM UBERABA (MG): PRIMEIROS APONTAMENTOS
}

\author{
Stella Márcia de Mesquita Pereira ${ }^{(a)}$, Valéria Machado Emiliano ${ }^{(b)}$, Fábio de Oliveira Sanches \\ ${ }^{(c)}$, Ricardo Vicente Ferreira ${ }^{\text {(d) }}$
}

${ }^{(a)}$ Dep. de Geografia da Universidade Federal do Triângulo Mineiro,stellammpereira@gmail.com

(b) Dep. de Geografia da Universidade Federal do Triângulo Mineiro, valeriamachadoemiliano17@ gmail.com

${ }^{(c)}$ Dep. de Geografia da Universidade Federal do Triângulo Mineiro, fsanches.73@ gmail.com

(d) Dep. de Geografia da Universidade Federal do Triângulo Mineiro, rcrdvf@ gmail.com

\section{Eixo: Climatologia em diferentes níveis escalares: mudanças e variabilidades}

\begin{abstract}
Resumo
O aumento das temperaturas planetárias associadas aos gases do efeito estufa tem sido os responsáveis pela intensificação dos eventos climaticos extremos. O objetivo do trabalho foi analisar o comportamento dos eventos extremos de temperatura em Uberaba buscando evidências de mudanças climáticas. Foram utilizados dados diários da Estação do INMET de Uberaba no período de 19892015 buscando analisar a quantidade de noites quentes com temperatura mínima $>20^{\circ} \mathrm{C}$ (TR), o valor máximo mensal das temperaturas máximas diárias $\left(\mathrm{TX}_{\mathrm{X}}\right)$ e o valor máximo mensal das temperaturas mínimas diárias $\left(\mathrm{TN}_{\mathrm{X}}\right)$. A aplicação do Teste de Mann-kendall na tendência dos dados revelou que as temperaturas máximas das máximas e as máximas das mínimas apresentaram tendências signficativas para mudanças de comportamento. A tendência positiva das noites quentes (TR) também se demonstrou significativa para mudança climática. Sendo assim, os resultados encontrados para Uberaba corroboram com os resultados dos relatórios do IPCC sobre mundaças climáticas.
\end{abstract}

Palavras chave: mudanças climáticas, eventos extremos, testes estatísticos, Triângulo Mineiro, aquecimento global.

\section{Introdução}

Nos últimos anos, os efeitos das mudanças climáticas globais e suas respectivas repercussões em diversas escalas tornaram-se temas de diversos encontros científicos, sobretudo após a sequência de relatórios divulgados pelo Painel Intergovernamental sobre Mudanças Climáticas (IPCC em inglês).

A elevação das temperaturas planetárias associadas às emissões de gases do efeito estufa e o aumento dos eventos climáticos extremos (tempestades, chuvas extremas, estiagens prolongadas, ondas de calor/frio, por exemplo) norteiam diversos trabalhos produzidos por pesquisadores do mundo (VINCENT et al., 2005; HAYLOCK et al., 2006; ALEXANDER et al., 2006; OBREGÓN e MARENGO, 2007; SILLMANN \& ROECKNER 2008; BLAIN, 2010; MARENGO et al., 2012; VALVERDE \& MARENGO, 2014; CHOI et al., 2014; SHARMAA \& BALBEB, 2014; ASADIEH \& KRAKAUER, 2015; BARBU et al., 2015; RAO et al., 2015; PERKINS-KIRKPATRICK et al, 2016 entre outros). 
Considerando a temática dos estudos sobre mudanças climáticas, a Organização Meteorológica Mundial (OMM), criou em 1993 o CLIVAR (Grupo de Variabilidade e Previsibilidade do Sistema Oceanoatmosfera) com o objetivo de descrever e compreender os processos oceano-atmosfera responsáveis pela variabilidade climática e previsibilidade nas escalas de tempo sazonais, interanuais, decenais e centenárias. Seu trabalho constitui na coleta e análise de dados observados para o desenvolvimento e aplicação de modelos do sistema climático acoplado, em cooperação com outras instituições de pesquisa e atividades de observação (http://www.clivar.org/about/about-clivar).

O CLIVAR tem desenvolvido estudos referentes às mudanças climáticas analisando o comportamento extremo do conjunto de variáveis climáticas por meio do seu grupo de pesquisadores interinstitucionais denominado Expert Team onClimateChangeDetectionandIndices (ETCCDI) (WMO, 2012). Dessa forma, nos últimos anos, diversos estudos têm procurado identificar alterações ou mesmo variabilidades climáticas em escalas menores (níveis regional e local), dada à necessidade de se compreender aspectos pontuais nas mudanças.

Com base na metodologia proposta pelo ETCCDI, os trabalhos de Paiva e Clarke (1995), Marengo e Alves (2005), Vincent et al. (2005), Haylock et al. (2006), Alexander et al. (2006), Folhes e Fisch (2006); Obregón e Marengo (2007), Sillmann\&Roeckner (2008), Blain (2009, 2010), Alves et al. (2009) e Lima et al. (2009), Orlowsky\&Seneviratne (2012), Silva Dias et al. (2013), Sanches et al. (2013; 2014) Choi et al. (2014), Sharmaa\&Balbeb (2014), Rao et al. (2015), Perkins-Kirkpatrick et al. (2016) entre outros, têm procurado analisar o comportamento das variáveis (temperatura e precipitação) ao longo do tempo em diversas partes do globo.

Uma das principais ferramentas de análises desses trabalhos são os testes estatísticos não-paramétricos, principalmente o Teste de Mann-Kendall (SIEGEL, 1975).

O Teste de Mann-Kendall compreende um teste estatístico não-paramétrico para identificação de tendências, recomendado pela Organização Meteorológica Mundial (OMM) para estudos de tendências climáticas em séries temporais longas (PAIVA e CLARKE, 1995; GROPPO et al., 2001; MARENGO e ALVES, 2005; ALEXANDER et al., 2006; FOLHES e FISCH, 2006; OBREGÓN e MARENGO, 2007; BLAIN, 2010), sendo amplamente utilizado nos últimos anos em trabalhos que visem a verificação de ocorrência de mudanças climáticas (SANCHES, 2015).

Sendo assim, o objetivo do trabalho é a analisar o comportamento dos eventos extremos de temperatura diária na região de Uberaba, no período de 1989-2015, buscando evidências de mudanças climáticas. 


\section{Material e Método}

Para este trabalho foram utilizados dados de temperatura obtidos a partir do Banco de Dados Meteorológicos para Ensino e Pesquisa (BDMEP) do Instituto Nacional de Meteorologia (INMET) (http://www.inmet.gov.br/portal/index.php?r=bdmep/bdmep). Foram utilizados dados diários de temperatura do período de 1989-2015 da Estação Meteorológica de Superfície (Convencional) de Uberaba (OMM: 83577) (Figura 1).

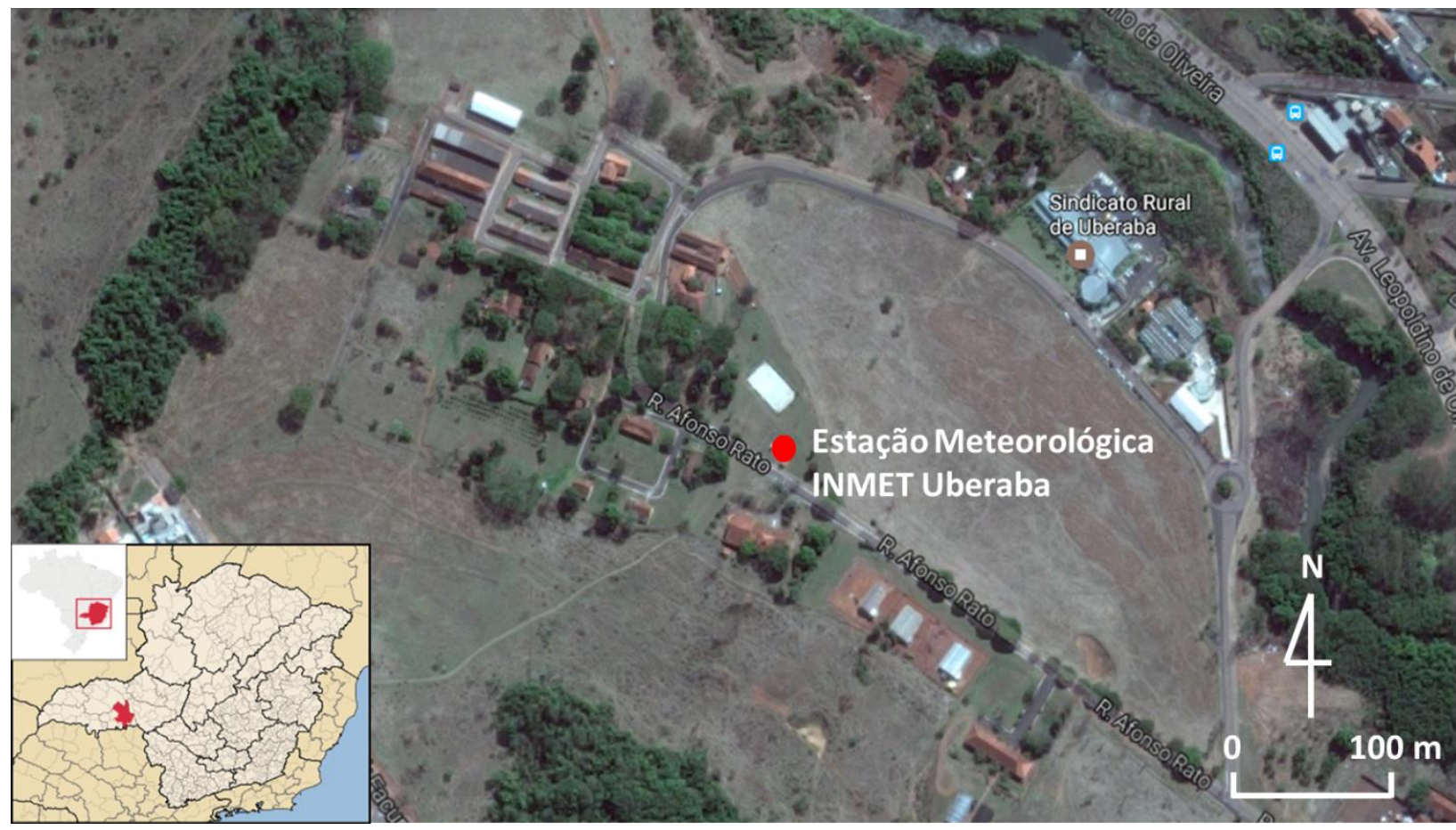

Figura 1 - Localização da Estação Meteorológica do INMET de Uberaba e sua área de entorno. Fonte: Adaptado de maps.google.com

A partir dos parâmetros metodológicos propostos pelo Expert Team onClimateChangeDetectionandIndices (ETCCDI) (http://etccdi.pacificclimate.org/list 27 indices.shtml) para a identificação de eventos extremos, foram identificados e analisados os parâmetros de temperatura descritos na Tabela 1.

Tabela I. Parâmetros pluviométricos para eventos extremos. 


\begin{tabular}{ll}
\hline Parâmetro & Descrição \\
\hline $\mathrm{TR}$ & Número de noitestropicais. Contagemanual dos dias com temperaturamínima \\
& superior a $20^{\circ} \mathrm{C}$. \\
$\mathrm{TX}_{\mathrm{X}}$ & Valor máximo mensal da temperaturamáximadiária \\
$\mathrm{TN}_{\mathrm{X}}$ & Valor máximo mensal da temperaturamínimadiária \\
\hline
\end{tabular}

Fonte: ETCDDI (http://etccdi.pacificclimate.org/list_27 indices.shtml)

Os dados de temperatura foram organizados em planilhas eletrônicas (Software Excel - Microsoft Inc) para serem extraídos os parâmetros de análise. Após sua obtenção, as tendências ao longo da série foram submetidas à avaliação qualitativa por meio da aplicação do teste de Mann-Kendall (MK), utilizando o software PAST (HAMMER et al., 2001) buscando evidências de mudança de comportamento que possam ser consideradas como mudanças climáticas.

O MK tem sido empregado em diversos trabalhos de pesquisa como objetivo de encontrar evidências de mudanças de comportamento que possam ser consideradas como evidências de possíveis mudanças climáticas (PAIVA e CLARKE, 1995; GROPPO et al., 2001; MARENGO e ALVES, 2005; ALEXANDER et al., 2006; FOLHES e FISCH, 2006; OBREGÓN e MARENGO, 2007; BLAIN, 2010; SANCHES et al., 2013; 2014 entre outros).

\section{Resultados e Discussão}

Os resultados da análise das temperaturas máximas das máximas mensais $\left(\mathrm{TX}_{\mathrm{x}}\right)$ pode ser observado na Figura 2. Verifica-se que, em todos os meses, que a tendência dos dados é positiva, ou seja, de aumento das temperaturas ao longo do período de 27 anos.

O resultado da aplicação do Teste MK (Tabela II) demonstra que a tendência de aumento das temperaturas máximas das máximas mensais $\left(\mathrm{TX}_{\mathrm{X}}\right)$ foi considerada significativa para mudança de comportamento em sete dos doze meses.

A Tabela II também mostra o resultado da aplicação do Teste MK para as temperaturas máximas das mínimas mensais (TNx). Nesse caso, a tendência positiva significativa para mudança de comportamento ocorreu em nove dos doze meses.

A tendência de aumento significativo dessas temperaturas foi verificada nos meses de verão (dezembro, janeiro, fevereiro), outuno (março e maio), inverno (junho, julho e agosto) e na primavera (outubro e dezembro).

Dessa forma, não é possível atribuir a elevação das temperaturas apenas a estação quente do ano. A tendência de elevação das temperaturas é comum ao longo dos meses no ano. 

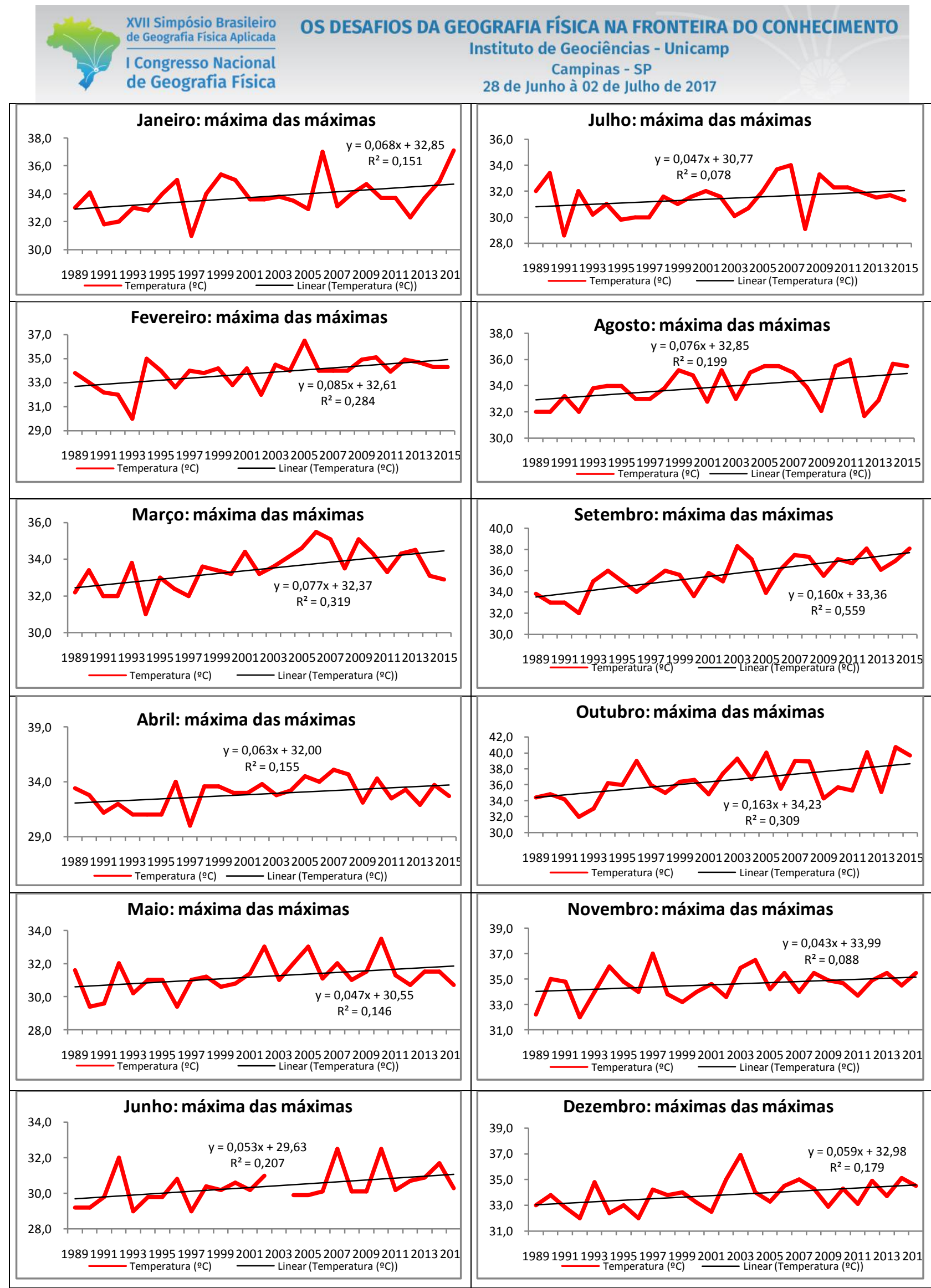

Figura 2-Tendência linear das temperaturas máximas das máximas mensais - Uberaba (MG). 
Tabela II - Resultado da aplicação do Teste de Mann-Kendall para as temperaturas em Uberaba (MG)

\begin{tabular}{lcc}
\hline \multirow{2}{*}{ Meses } & \multicolumn{2}{c}{ Escore (z) do Teste de Mann-Kendall } \\
\cline { 2 - 3 } & $\begin{array}{c}\text { Temperatura máxima das } \\
\text { máximas mensais }\end{array}$ & $\begin{array}{c}\text { Temperatura máxima das } \\
\text { mínimas mensais }\end{array}$ \\
\hline Janeiro & 1,57 & $\mathbf{2 , 7 7 ^ { * }}$ \\
Fevereiro & $\mathbf{2 , 8 6 *}$ & $\mathbf{2 , 9 4 *}$ \\
Março & $\mathbf{2 , 5 9 *}$ & $\mathbf{3 , 3 9 *}$ \\
Abril & 1,52 & 1,64 \\
Maio & 1,78 & $\mathbf{2 , 6 4 *}$ \\
Junho & $\mathbf{2 , 8 1 *}$ & $\mathbf{3 , 2 7 *}$ \\
Julho & 1,26 & $\mathbf{2 , 5 4}$ \\
Agosto & $\mathbf{2 , 6 0 *}$ & 1,32 \\
Setembro & $\mathbf{3 , 9 5 *}$ & $\mathbf{4 , 1 8 *}$ \\
Outubro & $\mathbf{2 , 8 2 *}$ & $\mathbf{3 , 3 9 *}$ \\
Novembro & 1,17 & 1,93 \\
Dezembro & $\mathbf{2 , 4 0 *}$ & $\mathbf{4 , 3 9 *}$ \\
\hline
\end{tabular}

*Significância em p-value $=0,05$.

Outro parâmetro analisado neste trabalho buscando encontrar evidências de aumento significativos das temperaturas em Uberaba corresponde a quantidade de noites com temperaturas superiores a $20^{\circ} \mathrm{C}$ (Figura $3)$.

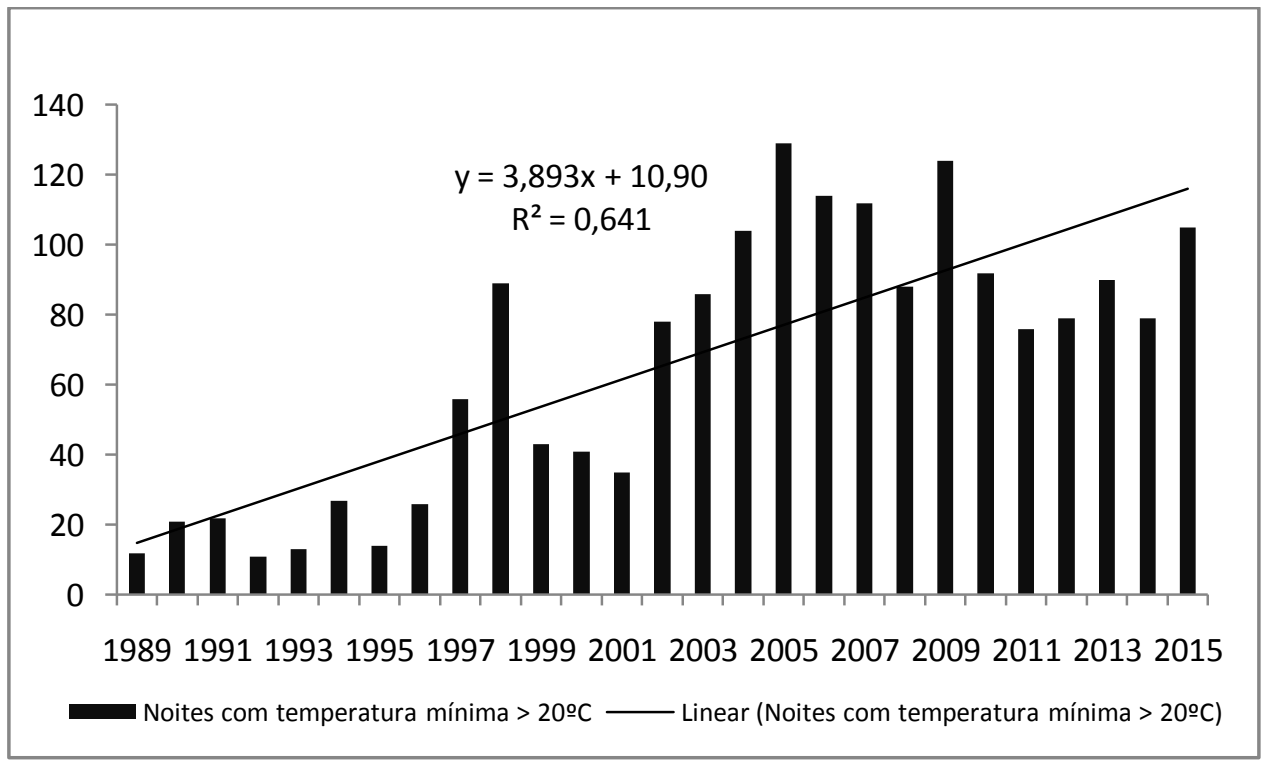

Figura 3 - Tendência das noites tropicais em Uberaba: quantidade de noites com temperatura mínima superior a $20^{\circ} \mathrm{C}$. 
A Figura 3 demonstra a quantidade de noites com temperaturas acima dos $20^{\circ} \mathrm{C}$ e a tendência anual desses dias nos 27 anos da série estudada.

Observa-se um salto inicial na quantidade dessas noites nos anos de 1997 e 1998. No entanto, a partir do ano de 2002 a quantidade dessasnoites atinge outro patamar em relação ao início da série. Verifica-se que houve um aumento médio de 101 dias com noites quentes (TR) ao longo da série estudada. Ao se avaliar a tendência da quantidade de noites quentes para Uberaba, o Teste de MK mostrou-se, também, significativo para mudança de comportamento $(\mathrm{Z}=4,23)$.

Tendo em vista que a Estação Meteorológica de Superfície do INMET de Uberaba (Figura 1) mantém no seu entorno as características técnicas de uso, ocupação e amortecimento recomendadas pela Organização Meteorológica Mundial (WMO, 1984),sendo assim, é possivel descartar a influência direta do tecido urbano na elevação das temperaturas locais e atribuir as tendências de aumento observadas neste trabalho a um fenômeno de maior escala (mudanças no tipo de uso e ocupação regional pela agricultura, supressão de vegetação nativa, nos arredores etc.).

\section{Considerações Finais}

A partir dos dados analisados no presente trabalho é possível concluir que:

1). Há uma tendência de elevação das tempetaturas máximas das máximas e das temperaturas maximas das mínimas mensais em Uberaba ao longo da série de dados analisados;

2). Essa tendência de aumento das temperturas máximas e das mínimas é observada ao longo de todos os meses;

3) O teste de MK demonstrou que a maior parte das tendências de aumento das temperaturas (maximas e mínimas) mensais podem ser consideradas como significativas para mudança de comportamento;

4). $\mathrm{O}$ aumento na quantidade de noites quentes ao ano (temperaturas mínimas superiores a $20^{\circ} \mathrm{C}$ ) foi igualmente considerado significativo, indicando mudança de comportamento.

Sendo assim, os estudos, ainda que preliminares, revelam fortes indícios de que as temperaturas em Uberaba (MG) estejam se elevando de acordo com a tendência observada para outas localidades no mundo, de acordo com os dados dos relatórios do IPCC. 


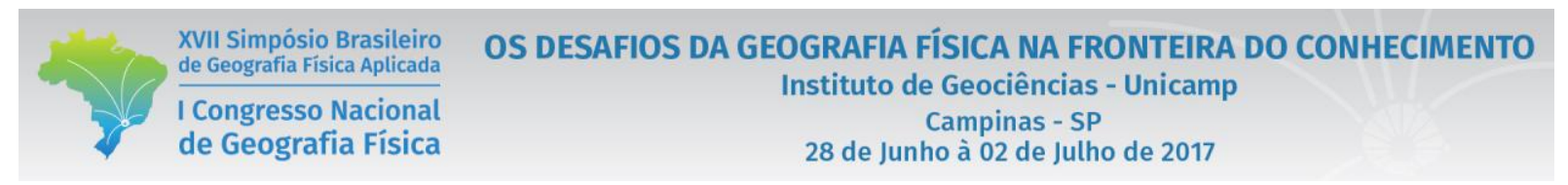

\section{Agradecimentos}

Os autores agradecem a Pró-reitoria de Pesquisa de Pós-graduação da UFTM e ao CNPQ pela bolsa de Iniciação Científica do edital No: 05/2016/PROPPG/UFTM/CNPQ para a primeira autora e ao auxílio à pesquisa para a segunda autora, ambas sob orientadação do terceiro autor.

\section{Bibliografia}

ALEXANDER, L.V; ZHANG, X.; PETERSON, T.C.; CAESAR, J.; GLEASON, B.; KLEIN TANK, A.M.G.; HAYLOCK, M.; COLLINS, D.; TREWIN, B.; RAHIMZADEH, F.; TAGIPOUR, A.; RUPA KUMAR, K.; REVADEKAR, J.; GRIFFITHS, G.; VICENT, L.; STEPHENSON, D. B.; BURN, J.; AGUILAR, E.; TAYLOR, M.; NEW, M.; ZHAIN, P.; RUSTICUCCI, M.; VAZQUEZ-AGUIRRE, J.L. Global observed changes in daily climate extremes of temperature and precipitation. Journal of Geophysical Research. vol. 111, D05109, 2006. (doi: 10.1029/2005JD00690).

ALVES, L.M.; MARENGO, J. Assessment of regional seasonal predictability using the PRECIS regional climate modeling system over South America. TheoreticalandAppliedClimatology, 100:337-350, 2009.

ASADIEH, B.; KRAKAUER, N.Y. Global trends in extreme precipitation: climate models versus observations. Hydrology and Earth Systems Sciences, 19, 877-891, 2015. doi:10.5194/hess-19-877-2015.

BALEN, D. S.; SANCHES, F. O.; CHECHI, L. Evaluation of trends in rainfall in the Upper Uruguay in Rio Grande do Sul (Brazil) between 1957-2012. In: V Simpósio Internacional de Climatologia. Anais... Florianópolis. Interação Oceano-atmosfera: impactos climáticos no presente e cenários futuros, 2013.

BARBU, N., CUCULEANU, V., STEFAN, S. Investigation of the relationship between very warm days in Romania and large-scale atmospheric circulation using multiple linear regression approach. TheoreticalandAppliedClimatology. p.1-12, 2015 (doi: 10.1007/s00704-015-1579-7).

BLAIN, G.C. Considerações estatísticas relativas à oito séries de precipitação pluvial da Secretaria de Agricultura e abastecimento do estado de São Paulo. Revista Brasileira de Meteorologia, v.24, n.1, 12-23, 2009.

BLAIN, G.C. Detecção de tendências monótonas em séries mensais de precipitação pluvial no Estado de São Paulo. Bragantia, Campinas, v.69, n4, p.1027-1033, 2010.

CHOI, W.; TAREGHIAN, R.; CHOIC, J.; HWANGC, C. Geographically heterogeneous temporal trends of extreme precipitation in Wisconsin, USA during 1950-2006. International Journal of Climatology, 34: 2841-2852. 2014. DOI: $10.1002 /$ joc.3878.

COELHO, C.A.S.; OLIVEIRA, C.P.; AMBRIZZI, T.; REBOITA, M.S.; CARPENEDO, C.B.; CAMPOS, J.L.P.S.; TOMAZIELLO, A.C.N.; PAMPUCH, L.A.; CUSTÓDIO, M.S.; DUTRA, L.M.M. ROCHA, R.P.; REHBEIN, A. The 2014 southeast Brazil austral summer drought: regional scale mechanisms and teleconnections. Climate Dynamics, August p.1-15, 2015. DOI 10.1007/s00382-015-2800-1.

FOLHES, M.T.; FISCH, G. Caracterização climática e estudo de tendência nas séries temporais de temperatura do ar e precipitação em Taubaté (SP). Ambi-Ägua, Taubaté, v.1, n.1, p.61-71, 2006.

FRANK, B., SEVERO, D.; SILVA, H.S. Validade do preenchimento de falhas em séries temporais. In: V Congresso Brasileiro de Meteorologia. Anais... Rio de Janeiro, 1988.

GROPPO, J.D.; MILDE, L.C.E.; GUAMERO, M.E.; MORAES, J.M.; MARTINELLI, L.A. Análise de séries temporais de vazão e de precipitação na Bacia do Rio Piracicaba. Revista de Ciência \& Tecnologia. v.8, n.18, p.109-117, 2001.

HAYLOCK, M.R.; PETERSON, T.C.; ALVES, L.M.; AMBRIZZI, T.; ANUNCIAÇÃO, Y.M.T.; BAEZ, J.; BARROS, V.R; BERLATO, M.A.; BIDEGAIN, M.; CORONEL, G.; CORRADI, V.; GARCIA, J.; GRIMM, A.M.; KAROLY, D.; MARENGO, J.A.; MARINO, M.B.; MONCUNILL, D.F.; NECHET, D.; QUINTANA, J.; 
REBELLO, E.; RUSTICUCCI, M.; SANTOS, J.L.; TREBEJO, I.; VINCENT, L.A. Trends in Total and Extreme South American Rainfall in 1960-2000 and Links withSeaSurfaceTemperature. JournalofClimate, v.19, p.1490$1512,2006$.

IPCC - Technical Summary. In: Climate Change 2013: The Physical Science Basis. Contribution of Working Group I to the Fifth Assessment Report of the Intergovernmental Panel on Climate Change. Cambridge University Press, Cambridge, United Kingdom and New York, NY, USA, 2013.

HAMMER, Ø., HARPER, D.A.T., RYAN, P.D. PAST: Paleontologicalstatistics software package for educationand data analysis. PalaeontologiaElectronica 4(1): 9pp. 2001.

LIMA, J.P.R.; ALVES, J.M.B. Um estudo de downscaling dinâmico de precipitação intrasazonal acoplado a modelo chuva-vazão na bacia hidrográfica Alto-médio São Francisco. Revista Brasileira de Meteorologia, v.24, n.3, 323338,2009 .

MARENGO, J.A.; ALVES, L.M. Tendências hidrológicas da bacia do rio Paraíba do Sul. Revista Brasileira de Meteorologia, v.20, n.2, 215-226, 2005.

MARENGO, J.A.; CHOU, S.C.; KAY, G.; ALVES, L.M.; PESQUERO, J.F.; SOARES, W.R.; SANTOS, D.C.; LYRA, A.A.; SUEIRO, G.; BETTS, R.; CHAGAS, D.J.; GOMES, J.L.; BUSTAMANTE, J.F.; TAVARES, P. Developmentof regional future climatechangescenarios in South AmericausingtheEta CPTEC/HadCM3 climatechangeprojections: Climatologyand regional analyses for theAmazon, São Francisco andtheParana River Basins. Climate Dynamics, v.38, Issue 9-10. 2012. p.1829-1848.

MARENGO, J.A.; VALVERDE, M.C. Caracterização do clima no Século XX e Cenários de Mudanças de clima para o Brasil no Século XXI usando os modelos do IPCC-AR4. Revista Multiciência, edição n. 8 - maio, Mudanças Climáticas, Campinas: 2007.

OBREgón, G.; MAREnGO, J.A. Caracterização do clima no Século XX no Brasil: Tendências de chuvas e Temperaturas Médias Extremas. Relatório n ${ }^{\circ}$ 2. Ministério do Meio Ambiente. Secretaria de Biodiversidade e Florestas. Diretoria de Conservação da Biodiversidade. 2007.

OLIVEIRA, L. F. C.; FIORENZE, A.P.; MEDEIROS, A.M.M.; SILVA, M.A.S. Comparação de metodologias de preenchimento de falhas de séries históricas de precipitação pluvial anual. Revista Brasileira de Engenharia Agrícola e Ambiental. v.14, n.11, p.1186-1192, 2010.

ORLOWSKY, B.; SENEVIRATNE, S.I. Global changes in extreme events: regional and seasonal dimension. ClimaticChange, 110:669-696, 2012. DOI 10.1007/s10584-011-0122-9

PAIVA, E.M.C.D.; CLARKE, R.T. Análise de tendência de precipitação na Amazônia. Revista Brasileira de Meteorologia. n.10 (1/2), p.37-41, 1995.

PERKINS-KIRKPATRICK, S.E.; WHITE, C.J.; ALEXANDER, L.V.; BOSCHAT, G.; COWAN, T.; EVANS, J.P.; EKSTRÖM, M.; OLIVER, E.C.J.; PHATAK, A.; PURICH, A. Natural hazards in Australia: heatwaves. Climatic Change, 1-14, April, 2016. DOI 10.1007/s10584-016-1650-0

RAO, V. B.; FRANCHITO, S.H.; SANTO, C.M.E.; GAN, M.A. An update on the rainfall characteristics of Brazil: seasonal variations and trends in 1979-2011. InternationalJournalofClimatology, 2015. DOI: 10.1002/joc.4345.

SANCHES, F. O. O Geógrafo-Climatologista e as mudanças climáticas: uma proposta metodológica. Revista Equador, v. 4, p. 101-118, 2015.

SANCHES, F.O.; VERDUM, R.; FISCH, G. Estudo de tendência de chuvas de longo prazo. Rev. Ambiente \& Água, vol. 8 n. 3 Taubaté - Sep. / Dec. 2013.

SANCHES, F.O.; VERDUM, R.; FISCH, G. Preenchimento de falhas em série de dados pluviométricos de Uruguaiana (RS) e análise de tendência. In: XVII Congresso Brasileiro de Meteorologia. Anais...Gramado-RS. 2012.

SANCHES, F.O.; VERDUM, R.; FISCH, G. Tendência de longo prazo das chuvas diárias no Sudoeste do Rio Grande do Sul: os eventos extremos e a arenização. Revista Brasileira de Geografia Física, v. 7, p. 1100-1109, 2014. 
SHARMAA, D.; BABELB, M.S. Trends in extreme rainfall and temperature indices in the western Thailand. InternationalJournalofClimatology. 34: 2393-2407, 2014. DOI: 10.1002/joc.3846.

SIEGEL, S. Estatística não-paramétrica para as Ciências do Comportamento. McGraw-Hill, Brasil. 1975.

SILLMANN, J.; ROECKNER, E. Indices for extreme events in projections of anthropogenic climate change. ClimaticChange. 86:83-104, 2008. DOI 10.1007/s10584-007-9308-6.

SILVA DIAS, M.A.F.; DIAS, J.; CARVALHO, L.M.V.; FREITAS, E.D.; SILVA DIAS, P.L. Changes in extreme dailyrainfall for São Paulo, Brazil. ClimaticChange, 116:705-722, 2013. DOI: 10.1007/s10584-012-0504-7

TUCCI, C.E.M.(Org.) Hidrologia: ciência de aplicação. 4.ed., $1^{\text {a }}$ reimp. - Porto Alegre: Editora da UFRGS/ABRH, 2009.

VALVERDE, M.C.; MARENGO, J.A. Extreme rainfall indices in the hidrographic basins of Brazil. Open Journal of Modern Hidrology, v.4, n.1, p. 10-26. 2014.

VINCENT, L.A.; PETERSON, T.C.; BARROS, V.R; MARINO, M.B.; RUSTICUCCI, M.; CARRASCO, G.; RAMIREZ, E.; ALVES, L.M.; AMBRIZZI, T.; BERLATO, M.A.; GRIMM, A.M.; MARENGO, J.A.; MOLION, L.; MONCUNILL, D.F.; REBELLO, E.; ANUNCIAÇÃO, Y.M.T.; QUINTANA, J.; SANTOS, J.L.; BAEZ, J.; CORONEL, G.; GARCIA, J.; TREBEJO, I.; BIDEGAIN, M.; HAYLOCK, M.R.; KAROLY, D. Observed Trends in Indices of Daily Temperature Extremes in South America 1960-2000. JournalofClimate, v.18, p.5011-5023, 2005.

WMO - $\quad$ World $\quad$ MeteorologicalOrganization $\quad-\quad$ Disponível em <http://www.wmo.int/pages/prog/wcp/wcdmp/CA 3.php>. Acesso em 30 de abril de 2012.

WORLD METEOROLOGICAL ORGANIZATION. Guia de Practicas Hidrológicas: acquisition y proceso de dados. 4ed. N168, Genebra, 1984. 